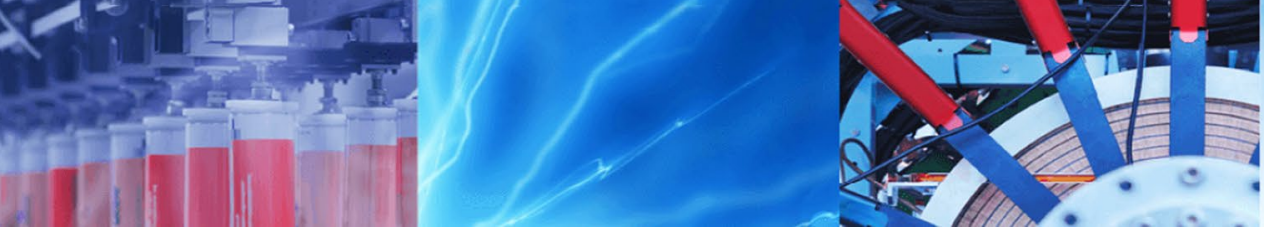

Research Article

\title{
FPGA implementation of r-FIFO-based high-speed data acquisition IOT architecture model
}

\author{
Sanskriti Gupta ${ }^{1}$ (1) $\cdot$ Mukul Sharma $^{2} \cdot$ Rashmi Chawla $^{1}$
}

Received: 26 August 2019 / Accepted: 4 March 2020 / Published online: 16 March 2020

(c) Springer Nature Switzerland AG 2020

\begin{abstract}
To enhance the utilization efficiency of Internet of Things (IoT) and cyber-physical system technologies, more collaboration in data transmission is recommended. These applications receive data from multiple sensors; hence, addition of efficient data acquisition system becomes mandatory. Past research works report use of data sharing among the tasks; however, this is only possible as long as the data meets the time-sensitive requirements of the tasks. To have an efficient communication between the different nodes of the loT applications, this research work proposes use of reconfigurable first-in, first-out ( $r$-FIFO) design to acquire data. This would not only reduce total sensing time but also the energy consumption, which is a conspicuous concernment in loT systems for the offline scenarios. This paper first provides an optimal algorithm for FIFO tasks in the offline case, and later the r-FIFO architecture is synthesized on the FPGA board using ZYBO (zynq- 7000). Further, the design is being implemented in SCL $180 \mathrm{~nm} \mathrm{CMOS} \mathrm{ASIC} \mathrm{technology.} \mathrm{Performance}$ measurement is performed by evaluating the frequency, total power, and the energy per bit of the design.
\end{abstract}

Keywords Internet of things · Data sharing · Algorithms

\section{Introduction}

With the advent of pervasive computing in 'Industry 4.0' $[1,2]$, the evolution of myriad Internet of Things (loT) technologies applications $[3,4]$ has emerged significantly. In an loT set-up, a number of sensors as data supplier and a central platform as the coordinator are customarily employed. Multiple applications apply their data requests to the platform. These requests needs to be stored before the consent of the sensed data is transmitted through the required platform. These distinct applications require big data acquisition system, analysis and display of global sensor data. For this connectivity of intelligent, instruments to evaluate strategic decisions have become inevitable [5]. The incoming data in the existing loT applications subsist in two categories (1) databases (2) sensing instruments, viz. sensors and computers. Currently, with different data types, access methods and communication protocols from various data sources, development of loT set-up to handle this input data becomes immensely complex. With mismatch in communication clock frequencies of various nodes, it is obvious that the set-up may not be able to fully exploit efficiency of sensors, energy, data, etc. This may impact overall efficiency of the system as it is incredible to have a different data format and uniform communication protocol for various instrument manufacturers.

For an loT set-up design, various research works have been reported. Most of the studies in past have focused on the overall application design [6-8] and communication protocol [9-11] in loT systems. There is no much research on handling variable big data at different clock frequencies. Tavakoli [12] proposes a data sharing algorithm for

Sanskriti Gupta, mailtosanskriti@gmail.com; Mukul Sharma, marvelmukulsharma@gmail.com; Rashmi Chawla, rashmichawlaymca@gmail.com | ${ }^{1}$ J.C. Bose University of Science and Technology, YMCA, Faridabad, India. ${ }^{2}$ Satyug Darshan Institute of Technology and Management, Faridabad, India. 
wireless sensor networks to minimize the complete sampling time. Here, the application used requests the need for discrete sample data at some point intervals, finally this data could be shared via multiple requests. Tavakoli [12] work was further improved by Fang et al. [13] by considering a continuous interval of sampling data by sending continuous sampling requests. In order to reduce the overall sampling time, an offline algorithm was developed. Also the overlapping of the data intervals could be shared by the other vacant requests. Zhao et al. [14] optimized the aggregate of min-max sensing time thus making fairness among the available sensors. Besides, this sufficient research has been made in the data acquisition system by implementing varied types of architectures. Kovac [15] proposed an interface between the general purpose interface bus and virtual instrument technology. This methodology provides comfort to access the available sensing instruments. Qiu et al. [16] proposed a data acquisition algorithm. Dynamic delay characteristics are analysed with the help of this algorithm. But this is appropriate for some specific sensing applications only and is not considerable for myriad data sources. The Open GIS (Geospacial) Consortium (OGC) [17] proposes a method for automatic integration of physical instruments. This is done with the use of Programmable Underwater Connector with Knowledge (PUCK) protocol. Although, many problems are solved with this development, but it also increases the cost of instrument manufactures. Zhu et al. [18] proposed sleep scheduling schemes for network systems to minimize energy consumption. But this work was not able to derive the complete benefit in terms of data sharing in the united/ collaborative sensing process. In brief, none of the aforementioned solutions can resolve comprehensively the issues with data acquisition of loT systems.

This paper focuses on the need of some generic solutions to overcome the frequency mismatch between the different nodes. These nodes in the data transmission work at the different operating frequencies and are further dependent on the application they are employed in. Here, the loT fog computing architecture is adopted, with three subsets (1) loT nodes (2) the cloud (3) Gateway layer (WAN-GSM, UMTS, MQTT, LTE, LTE-A; WiFi, Ethernet, Gateway control) [19-21]. The loT node constitutes a group of sensors data providers that can be designated as a central platform to perform loT operations. The loT gateways deployed works as message exchange between loT nodes and cloud. The solution proposed in this paper uses highspeed data acquisition (HSDA) algorithm for loT. Along with this a reconfigurable first-in, first-out (r-FIFO) design module is being implemented in the fog layer, keeping energy constraints as the main requisite. The r-FIFO module is further implemented on SCL $180 \mathrm{~nm}$ CMOS ASIC technology. This block could be used to stack the sensor data, and as a result, communication could be done in an efficient manner between the different nodes of the loT architecture.

In summary, the major contributions of this paper are as follows: (a) In the loT application scenario, HSDA parallel data acquisition technique is employed: Here, the global network of IoT, sensors and actuators are considered to be the main source of data. The massive, time-sensitive and heterogeneous nature of data accounts for myriad real-time challenges in decision-making scenarios. Thus, for any loT-based management system, the need of HSDA acquisition algorithm becomes requisite. (b) FPGA implementation of $r$-FIFO: The raw events of multiple sensors need to be aligned in time, so as to get high quality results. This growing number of various connected sensor devices makes the energy saving data acquisition process strenuous for any loT application. Hence, a FIFO interface is proposed to store the multiple data samples, thus relaxing its timing constraints issues. (c) SCL $180 \mathrm{~nm}$ CMOS ASIC technology implementation: This is done to verify real-time processing of the device that is mandatory, to be designed for various existing and necessary applications. The purpose of this research work is to reduce the development cost and improve the data acquisition efficiency, as data could be measured in the parallel manner with the use of $r$-FIFO. Rest of the paper is arranged as follows: Sect. 2 provides information about the HSDA and the reconfigurable FIFO design. System architecture for the loT applications is being proposed. Experimental results of the design r-FIFO for both the FPGA and ASIC implementation are given in Sect. 3. Section 4 provides the power result analysis of the design. Finally, Sect. 5 concludes the paper.

\section{Proposed system architecture for loT applications}

The loT applications have emerged in myriad sectors $[3,4]$. This research work employs a three-layered loT architecture and proposes the use ofr-FIFO design for low power and high latency communication. This proposal with various loT applications enables the exchange of data from the sensor nodes to control nodes through r-FIFO. This r-FIFO as a part of the fog computing layer communicates with multiple sensor nodes and gateway node. The employed sensor nodes are used to capture the relevant data from the environment, and later, these data are being used for further processing. The data are acquired using highspeed data acquisition (HSDA) [19]. The data acquired from sensor nodes are converted to digital format using analogto-digital converters (ADCs). Further, the data as per the requirement can be compressed/filtered using data computation algorithms. 
Figure 1 illustrates the complete proposed set-up. Here, the perception layer constitutes a group of sensors data providers that can be designated as a central platform to perform loT operations. It is flowed by HSDA to convert the physical sensor data to digital numeric value by sampling process. The data can be further stored in r-FIFO and process accordingly by management layer. Here, the gate way layer is present to communicate through a network to an enterprise back-end server that is running an loT platform. This helps to integrate the loT information into an existing enterprise and lastly the application layer provides the services requested by the customers.

The r-FIFO holds the final data acquired and can work in both the synchronous and asynchronous manner according to the application they are employed. The design of reconfigurable FIFO is inevitable as one requires the same clock frequency for local synchronization of the components present on the same chip module. In the case of inter-island communication (between the different chips), use of asynchronous mode in FIFO becomes necessary. The r-FIFO module mainly works as data storage and processing unit in the proposed design. Here, size of the FIFO is defined as depth $X$ depending on number of input bits, where the input bits are equal to the multiple data sample inputs from the various sensors. The data rate can be defined in terms of number of data inputs and time period of input data signal and the depth $X$ can be defined as Eq. (1):
Depth $X=\frac{\text { Difference (clk data rate) }}{\text { High frequency time period }}$

Difference $=$ Data rate of Fast clk (writeclk) - Data rate of Slow clk (readclk). The output of this block is fed to the microcontroller for further processing. This is the control node, which acts as a link between the gateway nodes, where the data are being put on the cloud for client's usage. The next subsections discuss in detail about the (A) HSDA (B) r-FIFO design

\section{A. High-speed data acquisition (HSDA)}

The parallel data acquisition is done using HSDA technique [16] in which measured sensor data are converted into digital numeric values as illustrated in Fig. 2. According to the

\begin{tabular}{|c|c|c|c|}
\hline Data Source & $\begin{array}{c}\text { Sensors } \\
\mathrm{S}_{1} \mathrm{~S}_{2} \mathrm{~S}_{3} \ldots \ldots \ldots \mathrm{S}_{\mathrm{j}}\end{array}$ & $\begin{array}{l}\text { Databases or } \\
\text { files }\end{array}$ & $\begin{array}{c}\text { Communication } \\
\text { Interface (RS232/ } \\
\text { Ethernet) }\end{array}$ \\
\hline $\begin{array}{c}\text { ASCII to binary } \\
\text { converter }\end{array}$ & $\mathrm{ADCs}$ & Con & trol Signals \\
\hline \multicolumn{4}{|c|}{ Standard Interface (i) } \\
\hline \multicolumn{4}{|c|}{ Data Acquisition Module with n interfaces } \\
\hline \multicolumn{4}{|c|}{ r-FIFO } \\
\hline \multicolumn{4}{|c|}{ Interface with Control Layer } \\
\hline \multicolumn{4}{|c|}{ Web Service MQTT etc. } \\
\hline
\end{tabular}

Fig. 2 HSDA architecture for parallel data acquisition
Fig. 1 The proposed loT architecture model

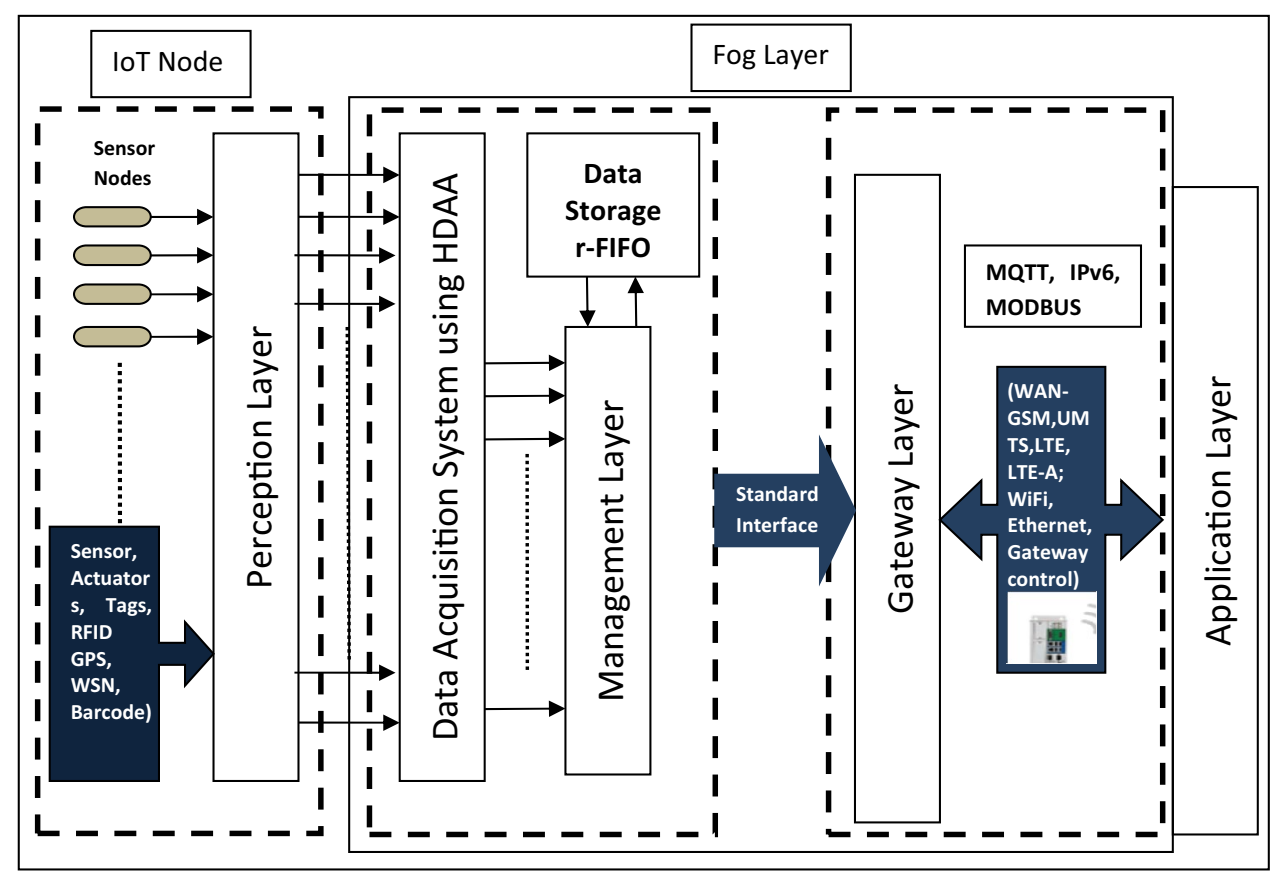

SN Applied Sciences A SPRINGER NATURE journa 
characteristics of different sensor data and data interface, the mapping relationship is established between sensor data and data interfaces which improves the data acquisition efficiency of loT. Data acquisition between different sensor data is independent of each other; hence, we can perform them in parallel.

Let $I_{i}$ denote interface information, where the index of interface and the sensor data information is denoted as $D_{j}$. Here' $j$ ' is the index of sensor data for each interface connection. The number of sensors connected to the $I_{i}$ interface is ' $j$ ' $=1,2, \ldots n$. As different data interfaces are independent from each other, we divide the data acquisition into $j$ rounds. And for each round, we acquire data from a data source on ' $l$ ' interfaces. There is a possible situation where no data source corresponds to the elements of matrix $M_{i, j}$. This represents the data source information of each accessed interface, so we set that value ' 0 '. The output of the algorithm is acquired sensor data and acquisition time with matrix $M_{i, j}$ form the sensor data. This describes all the information of the data sources that are accessed as input.

Algorithm for HSDA

1. To acquire data for round $j=1$ to $n$ from sensor data source, do

2. for each interface $i=1$ to $m$ do

3. if $M_{i, j}$ is not zero, then acquisition main program sends the list to the acquisition module correspond to sensor data $M_{i, j}$

4. end

5. To obtain acquisition time for interface $i=1$ to $m$ do

6. if $M_{i, j}$ is not zero then acquisition modules collect data according to the communication protocol and record the time $t_{i, j}$ required for the data acquisition

7. if $M_{i, j}=0$ then time required is zero

8. end

According to the obtained acquisition time $t_{m^{*} n}$ we can calculate the time required to complete one round of data acquisition. The time required for each round of data acquisition is the maximum value of each column of matrix, namely $t_{j}=\max T_{i, j}$. The time $t$ required to complete a complete data acquisition is $\max T_{i, j}$ for all sensor data. The time $t_{1}$ of the serial data acquisition is sum of $T_{i, j}$ for ' $j$ ' sensor data and ' $i$ ' interface. Obviously, $t \leq t_{1}$, and the efficiency of data acquisition is improved significantly with the increasing number of data sources.

\section{B. Reconfigurable FIFO design}

Local data synchronization is handled by clock, whereas asynchronous methodology is required for inter-island communication. The synchronization of different islands operating with different frequencies is inevitable with the use of synchronous FIFO module. However, these FIFO are required for the switches that are working in same clock domain. Distribution of single global clock is one of the major bottlenecks in today's high-performance VLSI system. This is mainly due to the multi-cycle cross-chip signalling, process variability and power dissipation. Hence, for local data synchronization clock is required to be synchronous in nature while data are required to be handled asynchronously for the inter-island communication. There requires the need for the reconfigurable FIFO (r-FIFO) that could be used for both the purposes. Hence, here an r-FIFO design is being implemented using gray encoding technique, in which gray code is passed from the read domain to write domain. The model has an advantage over a conventional FIFO module because it could be operated in both synchronous and asynchronous modes at a particular instant of time as per the requirement of the application [20].

The operation designed could be done via sync/bi-sync signal connected to both write and read modules. In synchronous mode, read and write operation is performed on the same clock frequency and in asynchronous mode read and write operation is performed on different clock frequency. (In this case, write operation is done at $40 \mathrm{MHz}$ clock frequency and read operation at $50 \mathrm{MHz}$ clock frequency.) This model has great relevance in terms of the proposed loT design application, as FIFO states that the earlier arrival tasks will have the earlier deadlines. Figure 3 gives the brief architecture design of the reconfigurable FIFO.

Here, the FIFO memory block is a dual port RAM that is accessed by both the read and write clock domains. The sync_ $r 2 w$ is the synchronizer that is used to synchronize read to the write pointer, and sync_w2r also acts similarly with vice-a-versa synchronization. The FIFO wptr and full module are synchronous to the write clock domain, whereas rptr and the empty module are synchronous to read clock domain. Beside this, the working of each port is clearly explained in Table 1. The further analysis of the designed architecture is provided in the next sections.

\section{Design analysis of the reconfigurable FIFO}

This design of reconfigurable FIFO is being simulated using the Vivado tool version 2018.2. The verification of the designed RTL code is done on the FPGA board using ZYBO (zynq- 7000). The simulated code is finally synthesized using Synopsys Design Compiler [21], where the timing and power constraints are checked at the synthesis level only. Later on, the design is physically designed using Synopsys IC Compiler tool. This is an ASIC designer tool where a small chip for the reconfigurable FIFO is being designed. It is being done so that this small unit could also 
Fig. 3 Reconfigurable FIFO architecture

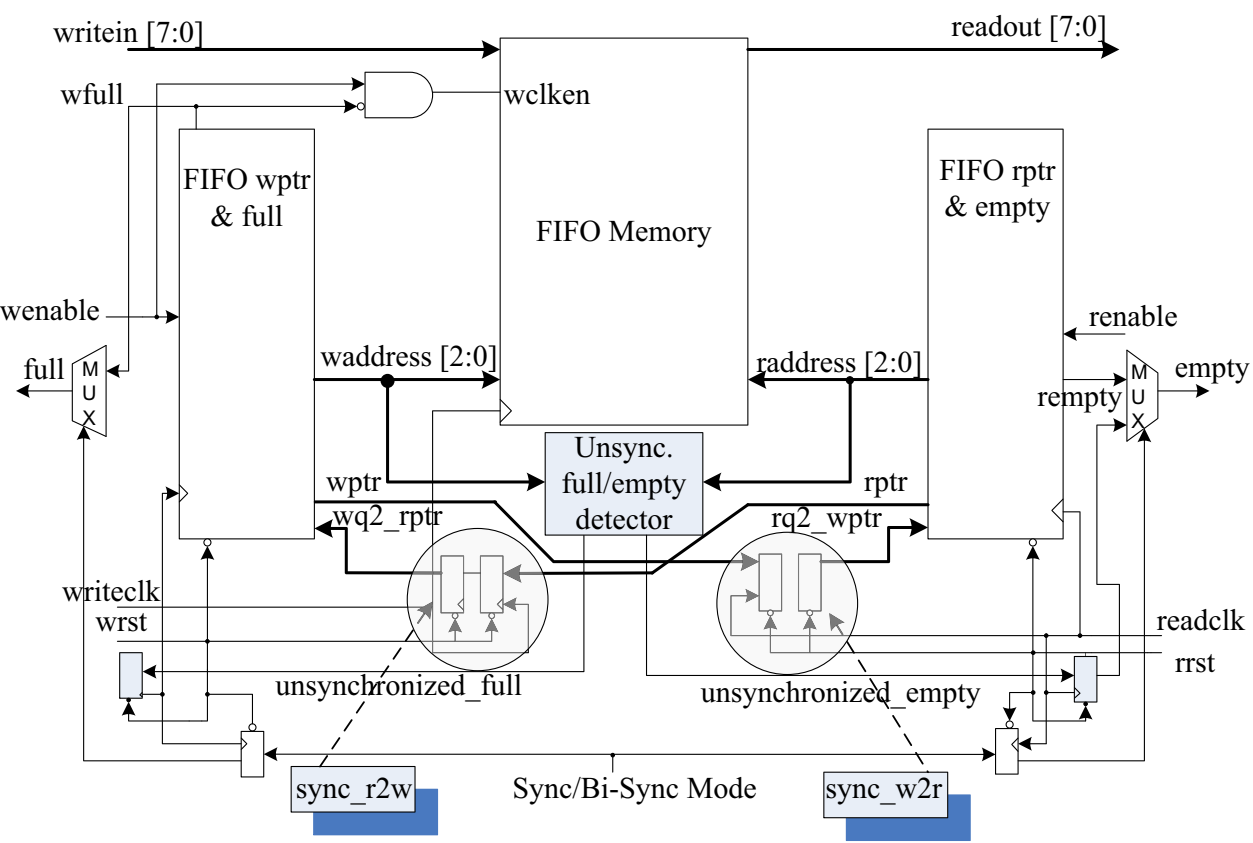

Table 1 Working of ports

\begin{tabular}{|c|c|c|c|}
\hline Port & Direction & No of bits & Importance \\
\hline writein & In & 8 & 8-bit data written into the FIFO \\
\hline readout & Out & 8 & 8-bit data read from the FIFO \\
\hline empty & Out & 1 & Represents empty state condition of the FIFO \\
\hline full & Out & 1 & Indicates no further data could be written in the FIFO, as the memory block is full \\
\hline writeclk & In & 1 & Write clock for the FIFO write operation \\
\hline readclk & $\ln$ & 1 & Read clock for the FIFO read operation \\
\hline wrst & In & 1 & Reset signal used to rest the write operation of the FIFO \\
\hline rrst & In & 1 & This is used to reset the read operation of the FIFO \\
\hline Sync/Bi-Sync & $\ln$ & 1 & $\begin{array}{l}\text { This signal decides that whether FIFO works in the synchronous mode or } \\
\text { asynchronous mode. If the signal is high, it works in a synchronous mode and } \\
\text { vice-a-versa in a low state }\end{array}$ \\
\hline
\end{tabular}

be used as a custom chip in more extensive system on chip (SoC) devices. The entire place and route flow are being done using SCL $180 \mathrm{~nm}$ CMOS technology [22]. It is a four metal layer process that works on the dual voltage power supply of $1.8 \mathrm{~V}$ for the core area and $3.3 \mathrm{~V}$ for the input and output (I/O) pads. The next subsections provide the simulation/verification and physical design implementation results, respectively.

\section{A. Vivado simulation and FPGA verification results}

As already explained in this paper, we have implemented the design of a dual port reconfigurable FIFO. The simulation result of the designed FIFO is provided in Fig. 4. It works on two different modes that are asynchronous and synchronous. The aided verification of the reconfigurable
FIFO design is done on Xilinx zynq Z-7000 series AP SoC/ FPGA board. Here, for testing the design of the reconfigurable FIFO frequency match between the LEDs of the I/O port and VGA (Video Graphics Array) port is done. The input is given by the push buttons, the control signal is provided by the switches, and the flag signal response is indicated by the LEDs present on the board. Initially, when the write operation is performed, led indicates that FIFO is empty. At this stage, data storage is done in the FIFO memory block with the use of a different combination of push buttons. When FIFO is in full state, then read operation is performed. To visibly see the stored data in the FIFO block, data are taken out from the unit designed using the VGA port present on the ZYBO (zynq-7000) board. Once the testing is compiled, further analysis of the designed 


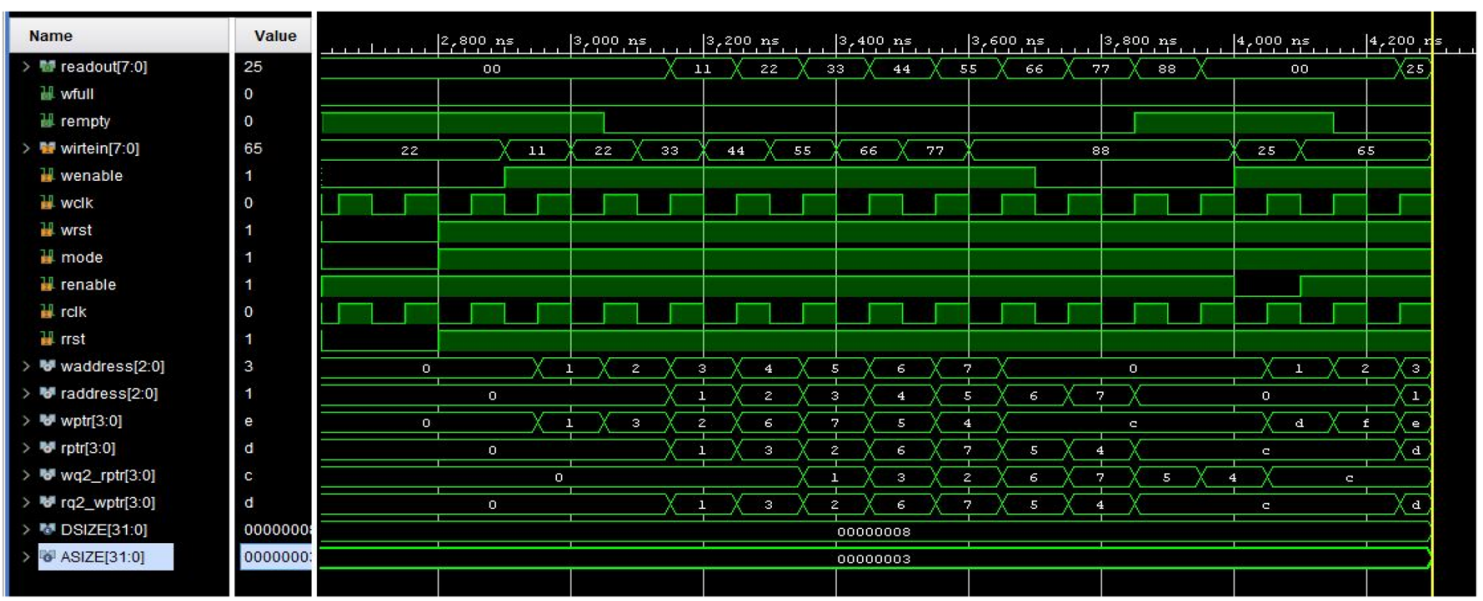

(a)

\begin{tabular}{|c|c|}
\hline Name & Value \\
\hline$>$ readout[7:0] & 00 \\
\hline 211 wiull & 0 \\
\hline 1213 rempty & 1 \\
\hline$>$ w wirtein[7:0] & 99 \\
\hline II wenable & 0 \\
\hline W wclk & 0 \\
\hline wrst & 0 \\
\hline W mode & 0 \\
\hline W renable & 1 \\
\hline 10 rclk & 0 \\
\hline II rst & 0 \\
\hline$>$ waddress[2:0] & 0 \\
\hline$>$ raddress[2:0] & 0 \\
\hline$>\boldsymbol{V}_{\text {wptr[3:0] }}$ & 0 \\
\hline$>W_{\text {rptr[3:0] }}$ & 0 \\
\hline$>\omega_{w q 2 \_ \text {rptr[3:0] }}$ & 0 \\
\hline$>$ rq2_wptr[3:0] & 0 \\
\hline > DSIZE[31:0] & 0000000 \\
\hline$>\sqrt[A S I Z E[31: 0]]{ }$ & 0000000 \\
\hline
\end{tabular}

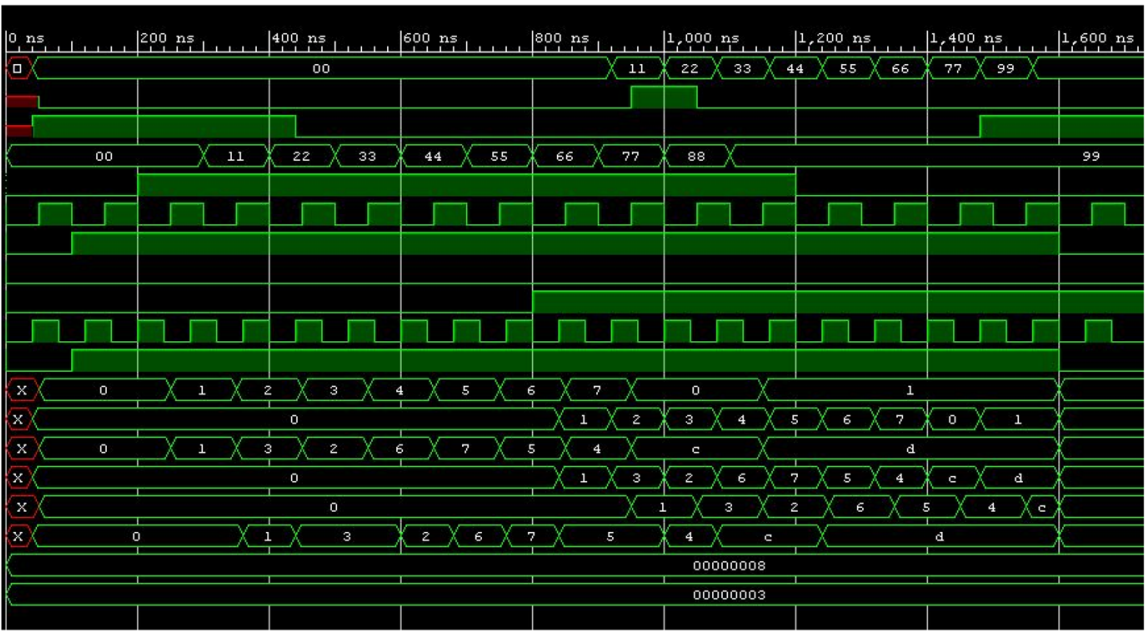

(b)

Fig. 4 a FIFO in synchronous mode b FIFO in asynchronous mode

Table 2 Design summary

\begin{tabular}{lclllc}
\hline Name & $\begin{array}{l}\text { Slice LUTs } \\
(17600)\end{array}$ & $\begin{array}{l}\text { Slice } \\
\text { registers } \\
(35200)\end{array}$ & $\begin{array}{l}\text { F7 } \\
\text { Muxes } \\
(8800)\end{array}$ & $\begin{array}{l}\text { Slice } \\
(4400)\end{array}$ & $\begin{array}{l}\text { LUT as } \\
\text { logic } \\
(17600)\end{array}$ \\
\hline FIFO (top) & 34 & 40 & 2 & 14 & 26 \\
fifomem & 8 & 8 & 0 & 3 & 0 \\
rptr_ & 13 & 8 & 1 & 7 & 13 \\
empty & & & & & \\
sync_r2w & 0 & 8 & 0 & 4 & 0 \\
sync_w2r & 0 & 8 & 0 & 4 & 0 \\
wptr_full & 13 & 8 & 1 & 5 & 13 \\
\hline
\end{tabular}

architecture is done. The lookup table (LUT) model provided by the design summary is shown as Table 2 .

Figure 4a shows the synchronous mode of the FIFO in which write clock (wclk) and read clock ( $r c l k)$ frequency are kept equal at $40 \mathrm{MHz}$. Whereas in Fig. 4b, asynchronous mode of FIFO is shown in which wclk is kept at $40 \mathrm{MHz}$ frequency and $r c l k$ is at $50 \mathrm{MHz}$ frequency. Here, the data in and out are represented by writein and readout signal, respectively.

\section{B. ASIC implementation results}

This physical designing process is done using the $\mathrm{SCL}$ (Semiconductor Laboratory) physical design kit. The environment of the tool could be set for three cases slow-slow (having the maximum transition time and delays), fast-fast (with minimum transition time and delays) and the typical condition. Here, the whole experimentation is being performed for the slow-slow corner set libraries, as this is the worst-case timing set. Then, the major concern of giving the design constraints to the reconfigurable FIFO is given by setting the operating frequency of the two different clock nets at $50 \mathrm{MHz}$ for readclk and $40 \mathrm{MHz}$ for writeclk, respectively.

\section{SN Applied Sciences}


Table 3 Standard cell count

\begin{tabular}{lccc}
\hline Module & Cell count & Area $\left(\mathrm{mm}^{2}\right)$ & NAND GEs \\
\hline Reconfigurable FIFO & 46 & & \\
Write full module & 46 & 0.0106 & \\
Read empty module & 8 & \\
Sync write to read & 8 & \\
Sync read to write & 219 & \\
FIFO memory & 2 & \\
Top level & &
\end{tabular}

Table 4 Power analysis

\begin{tabular}{lc}
\hline Constraints & Value $(\mu \mathrm{W})$ \\
\hline Cell internal power & 257.196 \\
Switching power & 53.842 \\
Leakage power & 0.155 \\
Total power & 311.039 \\
\hline
\end{tabular}

Here during the P\&R flow, core size and die size of the designed chip are taken to be the integer multiple of the tile width and tile height that are of 0.56 and 5.6 micron, respectively, of the standard cells available in the library. Besides, this special care is being taken, in order to meet the required timing and power constraints of the design. It is an iterative process and continues until the required voltage (IR) drop, and electromigration of the design analysis is met, according to the foundry specifications [23].The design is analysed at both the post-synthesis and postlayout level, where it meets all the violators. The standard cell count of the design summary could be clearly seen with the help of the provided Table 3.

Also, the slack that is the difference between the arrival time and the required time of the different clock nets is met [24]. The slack for the designed circuit is 2.98 and 3.57 for readclk and writeclk, respectively. Then, after performing the clock tree synthesis operation, the readclk takes 2 buffer levels, and writeclk acquires 3 buffer levels to meet the skew in the implemented design. The power analysis of the reconfigurable FIFO design is given in Table 4. The complete routed chip view of the design could be seen in Fig. 5.

\section{Results and discussions}

The architecture of reconfigurable FIFO was modelled in VHDL, and its simulation was performed on the Vivado software. The design is evaluated in terms of many parameters like area, slice count, timing, and power [25]. The synthesis of the design and its required ASIC flow is done, providing the proof of its verification. The post-synthesis power versus frequency curve when both the clocks operate in the synchronous mode is being illustrated in Fig. 6. The figure clearly explains that there is an increase in power with increase in the device frequency of the designed FIFO. This is because that with an increase in operating frequency there will be more switching in the
Fig. 5 Chip View of the Designed FIFO

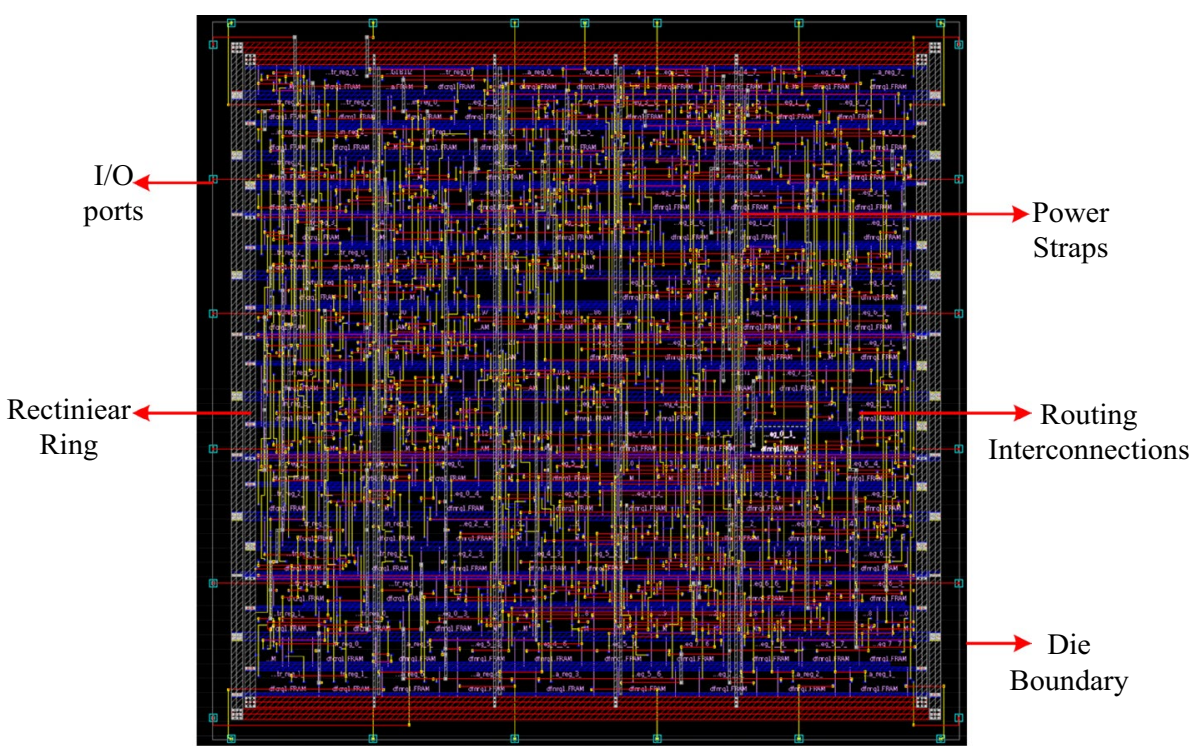

SN Applied Sciences A SPRINGER NATURE journal 


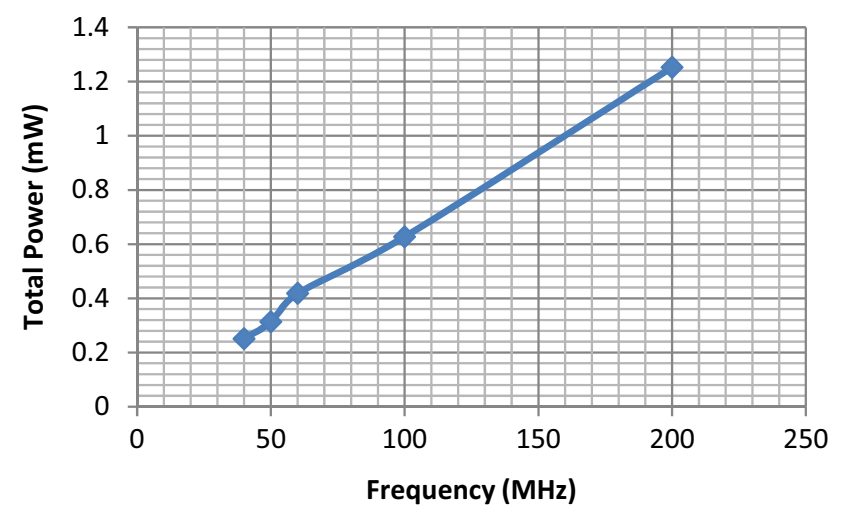

Fig. 6 Power versus frequency curve

device, thus affecting the efficiency of the power module. Hence, an appropriate frequency is chosen, according to the requirement of the application for the designed r-FIFO.

\section{Conclusion}

In this paper, we have investigated the data communication problem between the different nodes (working at different frequencies) of the loT architecture. The low latency is the main stumbling block for lot cloud computing applications. This implies the usage of front-end data calculation as in case of loT-based fog computing architecture. Further to address issues of frequency mismatch of various front-end devices or serially connected terminal devices and for the better collaboration between the platforms of the various loT applications, an idea of using the reconfigurable FIFO as a stack module is being proposed. The design of the FIFO module is being simulated, and its verification is done on the FPGA board using ZYBO. Besides this, the ASIC implementation of the block unit is also done, along with its power and timing consideration. This r-FIFO block has a total power dissipation of $311.09 \mu$ Wand it could also be used as a custom chip on large platforms. The energy per bit data for different clock domains of the designed reconfigurable FIFO is $0.777 \mathrm{pJ} /$ bit and $0.971 \mathrm{pJ} /$ bit for readclk and writeclk, respectively. In summary, the proposal can provide an optimum solution in fog computing to handle frequency inconsistency in various nodes.

\section{Compliance with ethical standards}

Conflict of interest On behalf of all the authors, the corresponding author states that there is no conflict of interest.

\section{References}

1. Vadiya S, Ambad P, Bhosle S (2018) Industry 4.0-a glimpse, vol 20. Elsevier, pp 233-238

2. Rojko A (2017) Industry 4.0 concept: background and overview. iJIM 11

3. Chawla R, Singal P, Garg AK (2018) A Mamdani fuzzy logic system to enhance solar cell micro-cracks image processing. 3D Res

4. Chawla R, Singhal P, Garg A (2019) Internet of things driven framework for smart solar energy system. ASME J Energy Resour Technol 142(1):011201-011201-9

5. Alavi AH, Jiao P, Buttlar WG, Lajnef B (2018) Internet of Thingsenabled smart cities: state-of-the-art and future trends. J Meas 129:589-606

6. Ning $H$, Wang $Z$ (2011) Future internet of things architecture: like mankind neural system or social orgainization framework? IEEE Commun Lett 461-463

7. Peng M, Li Y, Zhao Z, Wang C (2015) System architecture and key technologies for $5 \mathrm{G}$ heterogenous cloud radio access networks. In: IEEE Netw, pp 6-14

8. Riedel T, Fantana N, Genaid A, Yordanov D et al (2010) Using web service gateways and code generation for sustainable loT system development. In: IEEE, pp 1-8

9. Kelly et al (2013) Towards the implementation of loT foe environmental condition monitoring in homes. IEEE Sens 3846-3853

10. Gigli M, Koo SG (2011) Internet of things: services and applications categorization. Internet Things 27-31

11. Qiu Z et al (2016) loTI: internet of things instruments reconstruction model design. In: Instrumentation and measurement technology conference proceedings (I2MTC), pp 1-6

12. Tavakoli A, Kansal A, Nath S (2010) On-line sensing task optimization for shared sensors. In: The 9th ACM/IEEE international conference on information processing in sensor networks, pp 47-57

13. Fang X, Gao H, Li J, Li (2013) Application-aware data collection in wireless sensor networks. INFOCOM, 2013 Proceedings IEEE, pp 1645-1653

14. Zhao Q et al (2014) Fair energy-efficient sensing task allocation in participatory sensing with smartphones. IEEE, pp 1366-1374

15. Kovac VSK (2004) Virtual instrumentation and distributed measurement systems. J Electr Eng 50-56

16. Qui Z, Guo Z, Liu C (2012) Adaptive high-speed data acquisition algorithm in sensor network nodes. J Southeast Univ Nat Sci Ed 238-244

17. Toma DM, et al (2011) Smart sensors for interoperatable smart ocean environment. OCEANS 2011 IEEE, pp 1-4

18. Zhu C, Rodrigues JJPC, Leung VC, Shu L, Yang LT (2018) Trust based communication for industrial internet of things. IEEE Commun Mag 16-22

19. Qiu Z, Zhou Z, Qi S, Hu T (2019) HDAA: high-speed data acquisition algorithm of loT. Cognit Syst Signal Proc

20. Cummings CE (2002) Simulation and synthesis techniques for asynchronous FIFO design. In: Synopsys users group conference, San Jose

21. Synopsys (2011) Design compiler user guide

22. SCL (2018) SCL PDK of $180 \mathrm{~nm}$ technology

23. Synopsys (2015) IC compiler implementation user guide

24. Bhasker J, Chadha R (2009) Static timing analysis for nanometer designs a practical approach. Springer, New Providence

25. Synopsys (2010) Power compiler user guide. Synopsys, Inne

Publisher's Note Springer Nature remains neutral with regard to jurisdictional claims in published maps and institutional affiliations. 